\title{
Effects of whole-body vibrations on neuromuscular fatigue: A study with sets of different durations
}

\author{
Miloš Kalc ${ }^{\text {Corresp., } 1,2}$, Ramona Ritzmann ${ }^{3}$, Vojko Strojnik ${ }^{2}$ \\ ${ }^{1}$ Faculty of Medicine, Institute of Sports Medicine, University of Maribor, Maribor, Slovenia \\ 2 Faculty of Sport, Institute of Kinesiology, University of Ljubljana, Ljubljana, Slovenia \\ 3 Department of Biomechanics, Praxisklinik Rennbahn AG, Muttenz, Switzerland \\ Corresponding Author: Miloš Kalc \\ Email address: milos.kalc@ism-mb.si
}

Background. Whole body vibrations have been used as an exercise modality or as a tool to study neuromuscular integration. There is increasing evidence that longer WBV exposures (up to 10 minutes) induce an acute impairment in neuromuscular function. However, the magnitude and origin of WBV induced fatigue is poorly understood.

Purpose. The study aimed to investigate the magnitude and origin of neuromuscular fatigue induced by half-squat long-exposure whole-body vibration intervention (WBV) with sets of different duration and compare it to non-vibration (SHAM) conditions.

Methods. Ten young, recreationally trained adults participated in six fatiguing trials, each consisting of maintaining a squatting position for several sets of the duration of 30,60 or 180 seconds. The static squatting was superimposed with vibrations $\left(\mathrm{WBV}_{30}, \mathrm{WBV}_{60}, \mathrm{WBV}_{180}\right)$ or without vibrations $\left(\mathrm{SHAM}_{30}\right.$, $\mathrm{SHAM}_{60}, \mathrm{SHAM}_{180}$ ) for a total exercise exposure of 9-minutes in each trial. Maximum voluntary contraction (MVC), level of voluntary activation (\%VA), low- $\left(T_{20}\right)$ and high-frequency $\left(T_{100}\right)$ doublets, low-to-highfrequency fatigue ratio $\left(T_{20 / 100}\right)$ and single twitch peak torque $\left(T_{P T}\right)$ were assessed before, immediately after, then 15 and 30 minutes after each fatiguing protocol.

Result. Inferential statistics using RM ANOVA and post hoc tests revealed statistically significant declines from baseline values in MVC, $T_{20}, T_{100}, T_{20 / 100}$ and $T_{W_{P T}}$ in all trials, but not in \%VA. No significant differences were found between WBV and SHAM conditions. Magnitude based inference revealed a likely small to medium fatiguing effects in favour of $\mathrm{WBV}_{30}$ for MVC. Possibly small to likely moderate fatiguing effect in favour of $\mathrm{WBV}_{180}$ were observed for $\mathrm{TW}_{\mathrm{PT}}, \mathrm{T}_{20}$ and $\mathrm{T}_{20 / 100}$.

Conclusion. Our findings suggest that the origin of fatigue induced by WBV is not significantly different compared to control conditions without vibrations. The lack of significant differences in \% VA and the significant decline in other assessed parameters suggest that fatiguing protocols used in this study induced peripheral fatigue of a similar magnitude in all trials. However, trials with longer sets duration $\left(\mathrm{WBV}_{180}\right)$ were likely to induce a possibly larger magnitude of fatigue compared to SHAM condition. 


\section{Effects of whole-body vibrations on neuromuscular}

\section{2 fatigue: a study with sets of different durations}

3

4

5 Miloš Kalc ${ }^{1,2}$, Ramona Ritzmann ${ }^{3}$,Vojko Strojnik ${ }^{1}$

7

$8{ }^{1}$ Institute of Kinesiology, Faculty of Sport, University of Ljubljana, Ljubljana, Slovenia

92 Institute of Sports Medicine, Faculty of Medicine, University of Maribor, Maribor, 10 Slovenia

$11{ }^{3}$ Department of Biomechanics, Praxisklinik Rennbahn AG, Muttenz, Switzerland

15 Corresponding Author:

16 Miloš Kalc ${ }^{1,2}$

17 Taborska 8, Maribor, 2000, Slovenia

18 Email address: milos.kalc@ism-mb.si 


\section{Introduction}

20 Whole body vibration (WBV) transfers sinusoidal oscillations into the human body,

21

22

23

24

25

26

27

28

29

30

31

32

33

34

35

36

37

38

39

40

41

42

43

44

45

46

47

48

49

50

51

52

53

54

55

56

57

58

which inspired the use of this physical modality both as a tool to study the sensorimotor integration of the neuromuscular system and as an intervention stimulus with beneficial effects on performance (Rittweger, 2010). Early studies have suggested that short single sessions of WBV of 3 to 5 -minutes duration in a squat position immediately increase neuromuscular performance (Bosco et al., 2000; Cardinale \& Bosco, 2003), maximal voluntary contraction (MVC), jump performance and myoelectric activity (Alam, Khan \& Farooq, 2018). The acute increase in neuromuscular performance after vibration is referred to as 'post-activation potentiation' (PAP) for short-lasting enhancements (less than 1 minute) and as 'post-activation performance enhancement' (PAPE) for more extended performance enhancement periods lasting up to several hours (Blazevich \& Babault, 2019). Both phenomena are related to vibration-induced changes in the neuronal control of the affected skeletal muscles that encompass a facilitated central drive (Mileva, Bowtell \& Kossev, 2009; Krause et al., 2017) concomitant with modified reflexive activation at the spinal level (Rittweger, Beller \& Felsenberg, 2000; Ritzmann et al., 2018) persistent over a period of 15 minutes after vibration exposure (Krause et al., 2016; Ritzmann et al., 2018).

In everyday practice, therapists and practitioners promote longer WBV exposures (up to 10 minutes), although the effects of such exercise modalities are mostly unknown (Torvinen et al., 2002; Zory et al., 2013). By increasing the WBV stimuli duration up to a cumulative total of 4 to 10 minutes, it has been suggested that WBV may acutely induce fatigue rather than potentiation (Torvinen et al., 2002; de Ruiter et al., 2003; Erskine et al., 2007; Rittweger, 2010; Zory et al., 2013). For example, Torvinen et al. (2002) and de Ruiter et al. (2003) observed an immediate decrease of MVC after a 10×1-minute WBV intervention. However, no changes in MVC were observed in the control condition without vibrations. Even though various studies have reported a fatigue-induced drop in neuromuscular performance, there have been contradictory findings regarding the underlying mechanisms which favour either a central or peripheral origin. Several authors investigated the effect of WBV on central fatigue (Jordan et al., 2010; Maffiuletti et al., 2013; Zory et al., 2013) and were unable to find any difference in the level of voluntary activation (\%VA) between interventions with and without vibrations. To the best of our knowledge, the force-frequency fatigue-related mechanisms of WBVinduced peripheral fatigue have not been studied. By comparing the ratio of the electrically induced mechanical responses using low-frequency (below fusion frequency $-20 \mathrm{~Hz}$ ) and high-frequency (above fusion frequency $-100 \mathrm{~Hz}$ ) paired supramaximal electrical stimuli, peripheral fatigue can be subdivided into low- and high-frequency (Edwards, 2008; Millet et al., 2011). Analogous exercise-induced fatigue studies have demonstrated that prolonging exercise stimuli can shift the peripheral fatiguing mechanism towards low-frequency fatigue (Millet \& Lepers, 2004; Tomazin et al., 2012).

Peer) reviewing PDF | (2020:06:50532:1:0:NEW 2 Oct 2020) 
59 To better understand the intervention stimuli induced by WBV, it is crucial to establish

60 which fatiguing mechanisms occur after a single session of WBV, and how different

61 vibration parameters affect the magnitude and origin of neuromuscular fatigue. The

62 scientific and practitioner choices for WBV intervention are motivated by achieving high

63 superimposed effects throughout WBV to trigger physiological and neuromuscular

64 adaptations and thus, WBV parameters are combined accordingly (Abercromby et al.,

65 2007; Ritzmann, Gollhofer \& Kramer, 2013). Electromyography studies suggest that

66 side-alternating vibration exposure driven by high amplitude and frequency cause the

67 highest activation intensities in distal and proximal leg musculature (Abercromby et al.,

68 2007; Rittweger, 2010; Ritzmann, Gollhofer \& Kramer, 2013). In addition to vibration-

69 associated attributes, and in an analogy to strength training, the training load is mainly

70 determined by intensity and volume (Baechle \& Earle, 2008). Therefore, volume is

71 subdivided into number of set and repetitions with defined set duration (Campbell et al.,

72 2017). In a similar manner, vibration amplitude and frequency define the training

73 intensity in WBV interventions. However, to the best of our knowledge, there is a lack of

74 studies investigating how WBV intervention volume (set numbers and set duration)

75 affects the occurrence of neuromuscular fatigue.

76 Therefore, the aim of the present study was to investigate the magnitude and origin of

77 neuromuscular fatigue induced by long-exposure half-squat whole-body vibration

78 intervention (WBV) with sets of different duration and compare it with non-vibration

79 (SHAM) conditions. Thus, and with reference to, previous research involving long-

80 exposure WBV induced fatigue (Erskine et al., 2007; Zory et al., 2013) we selected a

81 long (cumulative exercise time of 9 minutes) static WBV fatiguing intervention divided

82 into sets of different duration ( $30 \mathrm{~s}, 60 \mathrm{~s}$ or $180 \mathrm{~s}$ ). In a series of MVC paradigms, we

83 applied different peripheral nerve stimulation techniques, allowing us to distinguish the

84 source of fatigue. We hypothesised that WBV exercise interventions would cause higher

85 magnitudes of fatigue compared to non-vibration intervention (Erskine et al., 2007; Zory

86 et al., 2013). We expected that fatigue magnitude would be dependent on the duration

87 of exposure and would increase with set-duration. We hypothesised that predominantly

88 peripheral, rather than central fatiguing mechanisms, would be causally involved

89 (Jordan et al., 2010; Maffiuletti et al., 2013; Zory et al., 2013).

90

91

92

93

94

95

96

97

98

\section{Materials \& Methods}

\section{Study design}

In a cross-over repeated measures design, each subject performed three different fatiguing exercise interventions with WBV and three exercise interventions in a sham condition without WBW (SHAM) to determinate the effect of WBV (Fig. 1A.). Each intervention comprised a cumulative exercise period with a duration of 9 minutes divided into different sets (either $18 \times 30 \mathrm{~s}$ or $9 \times 60 \mathrm{~s}$ or $3 \times 180 \mathrm{~s}$ ) with $120 \mathrm{~s}$ rest between sets 
102

103

104

105

106

107

108

109

110

111

112

113

114

115

116

117

118

119

120

121

122

123

124

125

126

127

128

129

130

131

132

133

134

135

136

(Fig 1A). The exercise interventions were performed on an activated vibration platform $\left(\mathrm{WBV}_{30}, \mathrm{WBV}_{60}, \mathrm{WBV}_{180}\right)$ and three on an inactive vibration platform $\left(\mathrm{SHAM}_{30}, \mathrm{SHAM}_{60}\right.$, $\mathrm{SHAM}_{180}$ ). Each intervention was executed on different visits with at least seven days rest in-between. The order was randomised. The subjects were not permitted to undertake explosive strength training or fatiguing workouts for 48 hours before each measuring day, in order to eliminate side-effects. The study design, materials and neuromuscular assessments are available for reference in protocols.io (dx.doi.org/10.17504/protocols.io.beadjaa6) Neuromuscular assessment in the resting position was performed at $t_{0}$ (baseline) prior to exercise intervention. The assessment consisted maximum voluntary contraction (MVC) of the knee extensors, interpolated with a high frequency ( $\left.T_{M V C}\right)$ twitch (10 ms interstimuli interval), followed $3 \mathrm{~s}$ later by a $100 \mathrm{~Hz}$ doublet $\left(T_{100}\right)$, followed $3 \mathrm{~s}$ later by a $20 \mathrm{~Hz}$ (50 ms interstimuli interval) doublet $\left(\mathrm{T}_{20}\right)$, and $3 \mathrm{~s}$ later by a potentiated single twitch (TW). The assessment procedure was executed according to (Millet et al., 2011) and repeated at 1 minute $\left(t_{f}\right)$, as well as at $15\left(t_{f 15}\right)$ and 30 minutes $\left(t_{f 30}\right)$ after the final 9minute intervention. All neuromuscular assessments were performed on the right leg.

\section{Subjects}

Ten healthy subjects ( 6 men and 4 women; age: $21.1 \pm 1.41$ years, mass: $77.8 \pm$ $11.73 \mathrm{~kg}$, BMI: $22.9 \pm 1.25$ ) volunteered to participate in the study. All subjects were recreationally trained athletes, participating in moderate endurance and strength training activities 3 times per week. Exclusion criteria were acute injuries in the upper or lower extremities, locomotor dysfunctions, pregnancy, cardiovascular or neurological conditions. All subjects signed the written informed consent and the study was approved by the Ethics Committee of the Faculty of Sport of the University of Ljubljana 975/2017 and conducted according to the Declaration of Helsinki II.

The sample size was estimated by means of a power analysis aiming to detect large effect sizes $(f=1.2$; alpha $=0.05$; power $=0.80)$.

\section{Intervention}

The interventions were performed on a side-alternating vibration platform (Galileo Fit, Novotec Medical, Germany) which was running at a frequency of $26 \mathrm{~Hz}$ (Rittweger, Mutschelknauss \& Felsenberg, 2003; Cochrane et al., 2010) and off, respectively, for WBV and SHAM conditions. Subjects were instructed to maintain a half-squat position with their knees flexed at an angle of $60^{\circ}$ (Ritzmann et al., 2010) for several sets with 2minute rest between sets. Kinematics were controlled with a goniometer. The subjects stood with their feet $40 \mathrm{~cm}$ apart at a point where the tilting platform reached peak-topeak displacement amplitude of $5 \mathrm{~mm}$ (Ritzmann, Gollhofer \& Kramer, 2013). 
137 At the beginning of each session, subjects underwent a 6-minute warm-up routine

138

139

140

141

142

143

144

145

146

147

148

149

150

151

152

153

154

155

156

157

158

159

160

161

162

163

164

165

166

167

168

169

170

171

172

173

174

175

176

consisting of bench stepping ( $20 \mathrm{~cm}$ high) at a frequency of $0.5 \mathrm{~Hz}$, swapping the leading leg at one minute intervals.

\section{Testing protocols}

During the neuromuscular assessment, the subjects remained seated in a custom-built isometric knee extension apparatus equipped with a force transducer (MES, Maribor, Slovenia) (Tomazin, Dolenec \& Strojnik, 2008; García-Ramos et al., 2016). The force transducer was calibrated prior to testing sessions. Each subject was seated in an upright position, hip at $100^{\circ}$ and trunk leaning against the backrest of the testing apparatus, fixed by straps over the pelvis and a horizontal pad over the distal third of the thigh. The knee joint axis was aligned with the mechanical axis of the dynamometer. The shin pad was placed just superior to the medial malleolus. The right knee joint was fixed at a $60^{\circ}$ angle $\left(0^{\circ}=\right.$ full extension) (Fig. 1C).

\section{Femoral nerve electrical stimulation}

The femoral nerve was stimulated by pressing a monopolar cathode $(10-\mathrm{mm}$ in diameter, Ag-AgCl, Type 0601000402, Controle Graphique Medical, Brie-ComteRobert, France) into the femoral triangle of the iliac fossa (Fig. 1C). A larger (102mm x $52 \mathrm{~mm}$, Compex, SA, Ecublens, Switzerland) self-adhesive electrode placed over the gluteal fold served as the anode. Electrical impulses (single, square wave, 1-ms duration) elicited by a high voltage constant current electrical stimulator (DS7A; Digitimer, Hertfordshire, UK) were used to trigger the muscle response, which was detected as a change in torque of the knee extensors. The stimulation intensity to elicit maximum knee extensors isometric twitch was determined in each subject at the beginning of each trial and maintained for the entire trial. Starting from an intensity of 10 $\mathrm{mA}$, the stimulation intensity was progressively increased by $10 \mathrm{~mA}$ until no further increase in torque was observed despite further increment in electrical current. The current at maximal twitch torque was additionally increased by a factor of 1.5 to obtain a supramaximal stimulus (Verges et al., 2009).

\section{Single twitch}

The torque change induced by a single supramaximal femoral nerve stimulus (Place et al., 2007) was analysed to obtain the peak torque value (TW $\left.\mathrm{PT}_{\mathrm{P}}\right)$.

\section{High- and low-frequency doublets}

The torque change induced by the paired high-frequency $(100 \mathrm{~Hz}$, i.e. $10-\mathrm{ms}$ interstimuli interval) and low-frequency ( $20 \mathrm{~Hz}$, i.e. 50 -ms interstimuli interval) supramaximal electrical stimuli (Place et al., 2007; Verges et al., 2009) was analysed to obtain the following parameters: peak torque from $100 \mathrm{~Hz}$ doublet $\left(T_{100}\right)$, peak torque from $20 \mathrm{~Hz}$ 
177 doublet $\left(T_{20}\right)$. In addition, the low- to high-frequency ratio $\left(T_{20 / 100}\right)$ was calculated using

178 the following formula:

179

$$
T_{20 / 100}=\frac{T_{20}}{T_{100}} * 100
$$

180

181

182

183

184

185

186

187

188

189

190

191

192

193

194

195

196

197

198

199

200

201

202

203

204

205

206

207

208

209

210

211

212

213

This ratio was used as a surrogate of low- to high-frequency tetanic stimulation (Verges et al., 2009).

\section{Maximal voluntary contraction with double twitch interpolated techniques}

Subjects were asked to perform a $5 \mathrm{~s}$ maximal isometric voluntary knee extension

(Verges et al., 2009). The signal was smoothed using a $0.5 \mathrm{~s}$ window moving average filter and peak torque (MVC) was retained for analysis. The double twitch interpolated technique (Allen, Gandevia \& McKenzie, 1995) was performed by superimposing a 100 $\mathrm{Hz}$ doublet on the isometric plateau ( $\left.\mathrm{T}_{\mathrm{MVC}}\right)$. A second analogous stimulation ( $\left.\mathrm{T}_{100}\right)$ on the relaxed muscle followed after 3 seconds (Fig. 1B). The ratio of the amplitude of the $\mathrm{T}_{\mathrm{MVC}}$ over $\mathrm{T}_{100}$ was then calculated to obtain the level of voluntary activation (\%VA):

$$
\% V A=\left(1-\frac{T_{M V C}-M V C}{T_{100}}\right) * 100
$$

\section{Statistics}

A two-way factorial ANOVA (Type III) was conducted in $\mathrm{R}(3.5 .1)$ with the afex package (Singmann et al., 2018) to compare the main effects of time $\left(t_{0}, t_{f}, t_{f 15}, t_{f 30}\right)$ and trial $\left(\mathrm{WBV}_{30}, \mathrm{WBV}_{60}, \mathrm{WBV}_{180}, \mathrm{SHAM}_{30}, \mathrm{SHAM}_{60}, \mathrm{SHAM}_{180}\right)$ and the interaction effect of time $\times$ trial. Generalised eta squared $\left(\eta_{G}^{2}\right)$ effect sizes were calculated for the ANOVA main and interaction effects. In the case of statistically significant interactions, post hoc comparisons with Sidak corrections were applied using the emmeans package (Lenth et al., 2018) in order to compare WBV and SHAM condition. Tukey-corrected pairwise post hoc tests were used to calculate differences to baseline within trials.

In addition to inference statistics, standardised changes in the mean of each measure were used to assess the magnitudes of effect (ES) between WBV and SHAM conditions

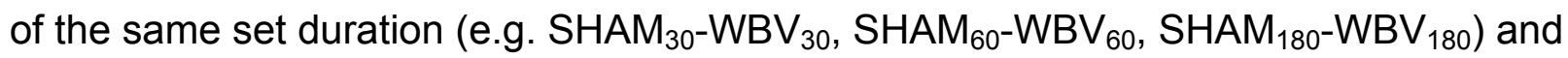
were then calculated using Cohen $\mathrm{d}$. The magnitude of ES was interpreted as follows: trivial $=<0.20$; small $=0.2-0.59 ;$ moderate $=0.60-1.19$; large $=1.20-1.99$; and very large $=>2.0$ based on recommendations by Hopkins (Hopkins et al., 2009).

Statistical significance was set at the level of $p<0.05$. ES results should be interpreted with caution, since negative values imply a larger fatiguing effect of WBV compared to SHAM condition and positive values imply a larger fatiguing effect for SHAM condition compared to WBV.

\section{Results}


215 Descriptive statistics for MVC and \%VA are displayed in Table 1; descriptive statistics

216 for $\mathrm{T}_{20}, \mathrm{~T}_{100}$ and $\mathrm{T}_{20 / 100}, \mathrm{TW}_{\mathrm{PT}}$ are listed in Table 2.

217

218 Maximum voluntary contraction

219 There was a statistically significant time effect $\left(F(3,27)=24.40, p<0.001, \eta_{G}^{2}=0.02\right)$,

220 but no significant trial effect $\left(F(5,45)=2.13, p=0.08, \eta_{G}^{2}=0.01\right)$ nor trial $x$ time

221 interaction effect $\left(F(15,135)=0.60, p=0.87, \eta_{G}^{2}=0.002\right)$ for MVC. Within-trial post hoc

222 tests showed differences between baseline and post-assessments (Fig. 2a).

223

224

225

226

227

228

229

230

231

232

233

234

235

\section{Level of voluntary activation (\%VA)}

There was a statistically significant time $\left(F(3,27)=3.67, p=0.024, \eta_{G}^{2}=0.02\right)$ and trial $\left(F(5,45)=2.52, p=0.042, \eta_{G}^{2}=0.08\right)$ effect, but no trial $x$ time interaction $(F(15,135)=$ $1,21, p=0.26, \eta_{G}^{2}=0.03$ ) for \%VA. Post hoc tests did not reveal significant differences between baseline and post-assessments (Fig. 2b).

\section{Peripheral fatigue}

There was a significant time effect $\left(F(3,27)=64.43, p<0.001, \eta_{G}^{2}=0.25\right)$ for $\mathrm{T}_{20}$. Trial effects $\left(F(5,45)=1.91, p=0.11, \eta_{G}^{2}=0.03\right)$ and trial $x$ time interaction effects $(F(15$, $\left.135)=0.90, p=0.56, \eta_{G}^{2}=0.007\right)$ remained statistically insignificant. Post hoc tests

revealed significant differences between baseline and post-assessments for each of the trials (Fig. 3a, Table 3).

236 There was a significant time effect $\left(F(3,27)=60.33, \mathrm{p}<0.001, \eta_{G}^{2}=0.15\right)$ for $\mathrm{T}_{100}$. Trial 237 effect $\left(F(5,45)=2.15, \mathrm{p}=0.07, \eta_{G}^{2}=0.03\right)$ and trial $x$ time interaction effect $(F(15,135)$ $\left.238=0.43, p=0.97, \eta_{G}^{2}=0.002\right)$ remained statistically insignificant. Post hoc tests revealed 239 significant differences between baseline and post-assessments for each of the trials

240 (Fig. 3b, Table 3).

241 There was a significant time effect $\left(F(3,27)=46.33, p<0.001, \eta_{G}^{2}=0.17\right)$ for T20/100.

242 Trial effect $\left(F(5,45)=1.06, p=0.40, \eta_{G}^{2}=0.02\right)$ and trial $x$ time interaction effect $(F(15$, $243135)=0.97, p=0.49, \eta_{G}^{2}=0.02$ ) remained statistically insignificant. Post hoc tests

244 revealed significant differences between baseline and post-assessments for each of the 245 trials (Fig. 3c, Table 3).

246

\section{Single twitch}

248 There was a significant time effect $\left(F(3,27)=48.80, p<0.001, \eta_{G}^{2}=0.23\right)$. Trial effects $249\left(\mathrm{~F}(5,45)=0.86, \mathrm{p}=0.52, \eta_{G}^{2}=0.006\right)$ and trial $\mathrm{x}$ time interaction effect $(\mathrm{F}(15,135)=$ $\left.2501.05, p=0.41, \eta_{G}^{2}=0.006\right)$ remained statistically insignificant for TW $\mathrm{PT}$. Post hoc tests 251 revealed significant differences between baseline and post-assessments for each of the 252 trials (Fig. 2c, Table 3). 


\section{Discussion}

254

255

256

The current study aimed to investigate the magnitude and origin of neuromuscular

257

258

259 fatigue induced by long-exposure half-squat whole-body vibration intervention (WBV) with sets of different duration and compare it with non-vibration (SHAM) conditions. Our findings revealed no superimposed effect of WBV compared to control conditions without vibrations.

260

261

262

\section{Maximal voluntary contraction}

263

Knee extensors MVC torque dropped by 7 to $12 \%$ after each fatiguing protocol, which is 264 in line with other WBV induced fatigue studies, where MVC torque decreased by approximately 8\% (de Ruiter et al., 2003; Erskine et al., 2007; Colson et al., 2009; Zory et al., 2013). Only Maffiuletti et al. (2013) reported a more substantial decline in MVC (266

267 $23 \%$ ), which is likely associated with the application of additional loads coupled with shorter inter-set rest periods compared to other studies and to our specific experimental

268

269 setting. This finding is in contrast with our hypothesis that longer set duration exercises superimposed with vibration ( $\mathrm{WBV}_{180}$ ) would produce greater fatigue compared to

270 $\mathrm{SHAM}_{180}$ condition. However, it has been previously suggested that potentiated

271 electrically elicited supramaximal doublets represent a more suitable indicator of

272

273 peripheral fatigue and contractile impairments compared to MVC torque (Place et al., 2007).

274

275

\section{Central fatigue}

276 The level of voluntary activation (\%VA) of the knee extensors was not significantly depressed by any intervention utilised in this study, which suggest that mechanisms located in the central nervous system (CNS) were not significantly involved in the

279 decline of MVC. These findings are in line with Colson et al. (2009) and Jordan et al.

280 (2010) but in contrast to de Ruiter et al. (2003) who reported a vibration-induced decline in knee extensors voluntary activation. Despite de Ruiter et al. (2003) reported a similar drop in \%VA compared to the present study (approx. 4\%), any difference in interpretation between the two studies could be biased by the lack of a control group or control condition in Ruiter et al. (2003) experiment coupled with the eligibility criteria for volunteers: in our study the population consisted of recreationally trained athletes and de Ruiter's et al. (2003) enrolled untrained students. Evidence exist for physiological differences and perquisites in motor control between sedentary and trained active subjects (Buford \& Manini, 2010). Being hypothesis-driven, our findings indicate that there are no evident superimposed effects of WBV on central fatiguing mechanisms compared to control conditions without WBV. This should be taken into consideration when designing exercise programs or research studies which intend to induce central 
292 fatigue. As such, WBV superimposed exercises are unlikely to be more effective than 293 maintaining a static squat alone.

294

295

296

297

298

299

300

301

302

303

304

305

306

307

308

309

310

311

312

313

314

315

316

317

318

319

320

321

322

323

324

325

326

327

328

329

330

\section{Peripheral fatigue}

To the best of our knowledge, this is the first study where electrically elicited supramaximal low- and high-frequency doublets were used to assess the origin and magnitude of peripheral fatigue after WBV exposure. For all protocols, $T_{20}$ was more affected than $\mathrm{T}_{100}$ leading to a decreased $\mathrm{T}_{20 / 100}$ ratio (Fig. 3c). These declines suggest the occurrence of low-frequency fatigue (LFF) in all trials. It is noteworthy that the $T_{20 / 100}$ ratio for SHAM interventions returned to baseline values 15 minutes after the intervention, while WBV interventions remained significantly depressed up to 30 minutes after the intervention. This suggests that LFF is stronger and more long-lasting when a WBV exercise is executed with an emphasis on exposing to longer sets of vibration. The observation favouring LFF as an underlying mechanism can additionally be supported by the findings obtained from single twitch data. Similar to $T_{20}$ and $T_{100}$, $\mathrm{TW}_{\mathrm{PT}}$ progressively decreased as the intervention continued.

\section{Underlying mechanisms}

The lack of difference between WBV and SHAM conditions observed in this study suggest that no beneficial effects on neuromuscular function exist when using superimposed WBV. This is particularly true for MVC and the level of voluntary activation. Even though some studies reported that WBV can induce modulation in the neuronal control, which is manifested as a facilitated central drive (Mileva, Bowtell \& Kossev, 2009; Krause et al., 2016) this does not translate into central fatigue. Furthermore, the decline in low- and high-frequency doublets, as well as single twitch torque, suggests that a mechanism underlying the decrease in force production in both WBV and SHAM treatments is an impairment in $\mathrm{Ca}^{2+}$ handling. This is followed by a gradual recovery of the $\mathrm{Ca}^{2+}$ depletion within the 15-30 min following WBV equal to the SHAM intervention. Underlying cellular fatiguing mechanisms explaining the results for SHAM and WBV may refer to three aspects (Westerblad et al., 2000; Allen \& Westerblad, 2001; Williams \& Ratel, 2009): a) since doublet peak torques progressively dropped at low- and high-frequencies of stimulation, there could be direct inhibition of inorganic phosphates $\left(\mathrm{P}_{\mathrm{i}}\right)$ on $\mathrm{Ca}^{2+}$, thereby producing an impairment in the cross-bridge force generation (Millar \& Homsher, 1990). However, it is unlikely that this mechanism alone accounts for low-frequency fatigue (Allen, Lannergren \& Westerblad, 1995). b) It is likely that the larger drop in $T_{20}$ compared to $T_{100}$ could indicate a precipitation in $\mathrm{Ca}^{2+}-\mathrm{P}_{\mathrm{i}}$ in the sarcoplasmic reticulum, leading to a decrease in free $\mathrm{Ca}^{2+}$ available for release (Allen \& Westerblad, 2001). In addition, c) reduced myofibrillar $\mathrm{Ca}^{2+}$ sensitivity can also affect force production (Bruton et al., 2008). Both mechanisms (b and c) have 
331 little impact on force production at high frequencies but a large effect on low frequencies

332 (Westerblad et al., 2000).

333

334

335

336

337

338

339

\section{Limitations}

The study might have some limitations. An important limitation of this study (similar to the majority of other vibration studies) is the lack of WBV load normalisation, as this may have considerable side-effects on the results, as was demonstrated by $\mathrm{Di}$ Giminiani et al. (2009). Another limiting aspect deals with different work/rest ratios between long sets ( $180 \mathrm{~s}$ work $-120 \mathrm{~s}$ rest) compared to other shorter set durations (30 $s-120 s$ and $60 s-120 s$ ). There is a great diversity in scientific and practitioner protocols and therefore, future studies should consider the variability in work/rest ratios and duration sets within the experimental design. Furthermore, the experiment was executed in the right leg only. The leg dominance has thereby not been considered as a variable of influence on fatigue and fatigue mechanisms.

\section{Conclusions}

The outcomes of this study suggest the origin of fatigue induced by half-squat with superimposed vibrations is no different from the control conditions without vibrations. Due to a lack of significant modulation of voluntary activation, it can be assumed that the fatiguing protocols used in this study predominantly affected peripheral mechanisms rather than central ones. The primary induced peripheral fatiguing mechanism seems to find its origin in low-frequency fatigue which most probably involves $\mathrm{Ca}^{2+}$ handling. The outcomes of this investigation seems to suggest that static squat with superimposed whole-body vibrations does not represent a larger fatiguing stimulus compared to static squat alone in recreationally active athletes.

\section{Acknowledgements}

362

363

364

\section{References}

365

Abercromby AFJ, Amonette WE, Layne CS, McFarlin BK, Hinman MR, Paloski WH. 2007. Vibration exposure and biodynamic responses during whole-body vibration training. Medicine and science in sports and exercise 39:1794-1800. DOI: 10.1249/mss.0b013e3181238aOf. 
369 Alam MM, Khan AA, Farooq M. 2018. Effect of whole-body vibration on neuromuscular 370 performance: A literature review. Work 59:571-583. DOI: 10.3233/WOR-182699.

371 Allen GM, Gandevia SC, McKenzie DK. 1995. Reliability of measurements of muscle strength and voluntary activation using twitch interpolation. Muscle \& Nerve 373 18:593-600. DOI: 10.1002/mus.880180605.

374 375

376

377

378

379

380

381

382

383

384

385

386

387

388

389

390

391 activity: cellular mechanisms of fatigue. Experimental Physiology 80:497-527. DOI: 10.1113/expphysiol.1995.sp003864.

Allen DG, Westerblad H. 2001. Role of phosphate and calcium stores in muscle fatigue. The Journal of Physiology 536:657-665. DOI: 10.1111/j.1469-7793.2001.t01-100657.x.

Baechle TR, Earle RW. 2008. Essentials of Strength Training and Conditioning. Human Kinetics.

Blazevich AJ, Babault N. 2019. Post-activation Potentiation Versus Post-activation Performance Enhancement in Humans: Historical Perspective, Underlying Mechanisms, and Current Issues. Frontiers in Physiology 10. DOI: 10.3389/fphys.2019.01359.

Bosco C, lacovelli M, Tsarpela O, Cardinale M, Bonifazi M, Tihanyi J, Viru M, De Lorenzo A, Viru A. 2000. Hormonal responses to whole-body vibration in men. European journal of applied physiology 81:449-454. DOI: $10.1007 / \mathrm{s} 004210050067$.

Bruton JD, Place N, Yamada T, Silva JP, Andrade FH, Dahlstedt AJ, Zhang S-J, Katz A, Larsson N-G, Westerblad H. 2008. Reactive oxygen species and fatigue-induced 
392

393

394

395

396

397

398

399

400

401

402

403

404

405

406

407

408

409

410

411

412

prolonged low-frequency force depression in skeletal muscle fibres of rats, mice and SOD2 overexpressing mice. The Journal of Physiology 586:175-184. DOI: 10.1113/jphysiol.2007.147470.

Buford TW, Manini TM. 2010. Sedentary individuals as "controls" in human studies: The correct approach? Proceedings of the National Academy of Sciences of the United States of America 107:E134. DOI: 10.1073/pnas.1008118107.

Campbell BI, Bove D, Ward P, Vargas A, Dolan J. 2017. Quantification of Training Load and Training Response for Improving Athletic Performance. Strength \& Conditioning Journal 39:3-13. DOI: 10.1519/SSC.0000000000000334.

Cardinale M, Bosco C. 2003. The use of vibration as an exercise intervention. Exercise and sport sciences reviews 31:3-7. DOI: 10.1097/00003677-200301000-00002.

Cochrane DJ, Stannard SR, Firth EC, Rittweger J. 2010. Acute whole-body vibration elicits post-activation potentiation. European Journal of Applied Physiology 108:311-319. DOI: 10.1007/s00421-009-1215-2.

Colson SS, Petit P-D, Hébreard L, Tessaro J, Pensini M. 2009. Whole body vibration does not enhance muscle activation. International journal of sports medicine 30:841-844. DOI: 10.1055/s-0029-1234082.

Di Giminiani R, Tihanyi J, Safar S, Scrimaglio R. 2009. The effects of vibration on explosive and reactive strength when applying individualised vibration frequencies. Journal of Sports Sciences 27:169-177. DOI:

$10.1080 / 02640410802495344$ 
413 Edwards RHT. 2008. Human Muscle Function and Fatigue. In: Ciba Foundation

$414 \quad$ Symposium 82 - Human Muscle Fatigue: Physiological Mechanisms. John Wiley $415 \quad$ \& Sons, Ltd, 1-18.

416 Erskine J, Smillie I, Leiper J, Ball D, Cardinale M. 2007. Neuromuscular and hormonal responses to a single session of whole body vibration exercise in healthy young men. Clinical physiology and functional imaging 27:242-8. DOI: 10.1111/j.1475-

García-Ramos A, Tomazin K, Feriche B, Strojnik V, de la Fuente B, ArgüellesCienfuegos J, Strumbelj B, Štirn I. 2016. The Relationship Between the Lower-

422 Body Muscular Profile and Swimming Start Performance. Journal of Human Kinetics 50:157-165. DOI: 10.1515/hukin-2015-0152.

Hopkins WG, Marshall SW, Batterham AM, Hanin J. 2009. Progressive statistics for 425 studies in sports medicine and exercise science. Medicine and Science in Sports and Exercise 41:3-13. DOI: 10.1249/MSS.0b013e31818cb278.

Jordan M, Norris S, Smith D, Herzog W. 2010. Acute effects of whole-body vibration on 429 peak isometric torque, muscle twitch torque and voluntary muscle activation of the knee extensors. Scandinavian Journal of Medicine and Science in Sports 20:535-540. DOI: 10.1111/j.1600-0838.2009.00973.x.

Krause A, Gollhofer A, Freyler K, Jablonka L, Ritzmann R. 2016. Acute corticospinal and spinal modulation after whole body vibration. Journal of Musculoskeletal \& Neuronal Interactions 16:327-338.

434 Krause A, Schönau E, Gollhofer A, Duran I, Ferrari-Malik A, Freyler K, Ritzmann R. 435 2017. Alleviation of Motor Impairments in Patients with Cerebral Palsy: Acute 
Effects of Whole-body Vibration on Stretch Reflex Response, Voluntary Muscle Activation and Mobility. Frontiers in Neurology 8:416. DOI: 10.3389/fneur.2017.00416.

439 Lenth R, Singmann H, Love J, Buerkner P, Herve M. 2018. emmeans: Estimated Marginal Means, aka Least-Squares Means.

441 Maffiuletti NA, Saugy J, Cardinale M, Micallef J-P, Place N. 2013. Neuromuscular fatigue induced by whole-body vibration exercise. European Journal of Applied Physiology 113:1625-1634. DOI: 10.1007/s00421-013-2590-2.

444 Mileva KN, Bowtell JL, Kossev AR. 2009. Effects of low-frequency whole-body vibration on motor-evoked potentials in healthy men. Experimental Physiology 94:103116. DOI: 10.1113/expphysiol.2008.042689.

447 Millar NC, Homsher E. 1990. The effect of phosphate and calcium on force generation in glycerinated rabbit skeletal muscle fibers. A steady-state and transient kinetic

Millet GY, Lepers R. 2004. Alterations of neuromuscular function after prolonged running, cycling and skiing exercises. Sports medicine (Auckland, N.Z.) 34:10516.

Millet GY, Martin V, Martin A, Vergès S. 2011. Electrical stimulation for testing neuromuscular function: from sport to pathology. European journal of applied physiology 111:2489-2500. DOI: 10.1007/s00421-011-1996-y. central and peripheral fatigue after sustained maximal voluntary contraction of the quadriceps muscle. Muscle \& Nerve 35:486-495. DOI: 10.1002/mus.20714. 
459 Rittweger J. 2010. Vibration as an exercise modality: how it may work, and what its 460 potential might be. European journal of applied physiology 108:877-904. DOI:

461 10.1007/s00421-009-1303-3.

462 Rittweger J, Beller G, Felsenberg D. 2000. Acute physiological effects of exhaustive 463 whole-body vibration exercise in man. Clinical physiology (Oxford, England) 464 $20: 134-42$.

465

Rittweger J, Mutschelknauss M, Felsenberg D. 2003. Acute changes in neuromuscular 466

467

468 23:81-6.

469

470

471

472

473

474

475

476

477

478

479

480 excitability after exhaustive whole body vibration exercise as compared to exhaustion by squatting exercise. Clinical physiology and functional imaging

Ritzmann R, Gollhofer A, Kramer A. 2013. The influence of vibration type, frequency, body position and additional load on the neuromuscular activity during whole body vibration. European journal of applied physiology 113:1-11. DOI: $10.1007 / \mathrm{s} 00421-012-2402-0$.

Ritzmann R, Kramer A, Gruber M, Gollhofer A, Taube W. 2010. EMG activity during whole body vibration: motion artifacts or stretch reflexes? European Journal of Applied Physiology 110:143-151. DOI: 10.1007/s00421-010-1483-x.

Ritzmann R, Krause A, Freyler K, Gollhofer A. 2018. Acute whole-body vibration increases reciprocal inhibition. Human Movement Science 60:191-201. DOI: 10.1016/j.humov.2018.06.011.

de Ruiter CJ, van der Linden RM, van der Zijden MJ a, Hollander AP, de Haan A. 2003. Short-term effects of whole-body vibration on maximal voluntary isometric knee 
extensor force and rate of force rise. European journal of applied physiology

482 88:472-5. DOI: 10.1007/s00421-002-0723-0.

483 Singmann H, Bolker B, Westfall J, Aust F, Højsgaard S, Fox J, Lawrence MA, Mertens 484 U, Love J, Lenth R, Christensen RHB. 2018. afex: Analysis of Factorial 485 Experiments.

486

487

488

489

490

491

492

493

494

495

496

497

498

499

500

501 skiing. European journal of applied physiology 103:189-94. DOI:

Tomazin K, Dolenec A, Strojnik V. 2008. High-frequency fatigue after alpine slalom 10.1007/s00421-008-0685-y.

Tomazin K, Morin JB, Strojnik V, Podpecan A, Millet GY. 2012. Fatigue after short (100m), medium (200-m) and long (400-m) treadmill sprints. European Journal of Applied Physiology 112:1027-1036. DOI: 10.1007/s00421-011-2058-1.

Torvinen S, Kannu P, Sievänen H, Järvinen T a HTLNT a H, Pasanen M, Kontulainen S, Järvinen T a HTLNT a H, Järvinen M, Oja P, Vuori I, Sievanen H, Jarvinen TAL, Pasanen M, Kontulainen S, Jarvinen TAL, Jarvinen M, Oja P, Vuori I. 2002. Effect of a vibration exposure on muscular performance and body balance. Randomized cross-over study. Clinical physiology and functional imaging 22:145-52.

Verges S, Maffiuletti N a, Kerherve H, Decorte N, Wuyam B, Millet GY. 2009. Comparison of electrical and magnetic stimulations to assess quadriceps muscle function. Journal of applied physiology (Bethesda, Md. : 1985) 106:701-10. DOI: 10.1152/japplphysiol.01051.2007. 
502 Westerblad H, Bruton JD, Allen DG, Lännergren J. 2000. Functional significance of 503 Ca2+ in long-lasting fatigue of skeletal muscle. European Journal of Applied $504 \quad$ Physiology 83:166-174. DOI: 10.1007/s004210000275.

505 Williams C, Ratel S (eds.). 2009. Human Muscle Fatigue. London ; New York: $506 \quad$ Routledge.

507 Zory RF, Aulbrook W, Keir DA, Serresse O. 2013. Occurrence of fatigue induced by a 508 whole-body vibration session is not frequency dependent. Journal of strength 509 and conditioning research 27:2552-2561. DOI: 10.1519/JSC.0b013e31827fd730. 


\section{Figure 1}

Experimental design and settings.

(A) Experimental design comprising the fatiguing protocols for all six visits and the timeline of each visit. Neuromuscular function was assessed before $\left(t_{0}\right)$, immediately after $\left(t_{f}\right), 15\left(t_{f 15}\right)$ and $30\left(t_{330}\right)$ minutes after vibration intervention. An expanded view of exercise exposure representing the $\mathrm{WBV}_{60}$ protocol (nine sets of $60 \mathrm{~s}$ of vibration exercise with $120 \mathrm{~s}$ rest between sets) is presented in detail. (B) Example of a torque signal from the neuromuscular testing procedure. An expanded view of an interpolated twitch is presented in the dotted box. The neuromuscular testing procedure comprised MVC of the quadriceps muscle combined with different electrical stimulation methods to assess the level of voluntary activation - \%VA (via the interpolated double twitch technique), quadriceps twitch torques in response to paired electrical stimuli at $100 \mathrm{~Hz}\left(T_{100}\right)$ and at $20 \mathrm{~Hz}\left(T_{20}\right)$, as well as single twitch (TW). (C) Schematic of the position of the subject during the neuromuscular assessment. An expanded view of the femoral nerve stimulation point in the popliteal fossa is presented within the box. 

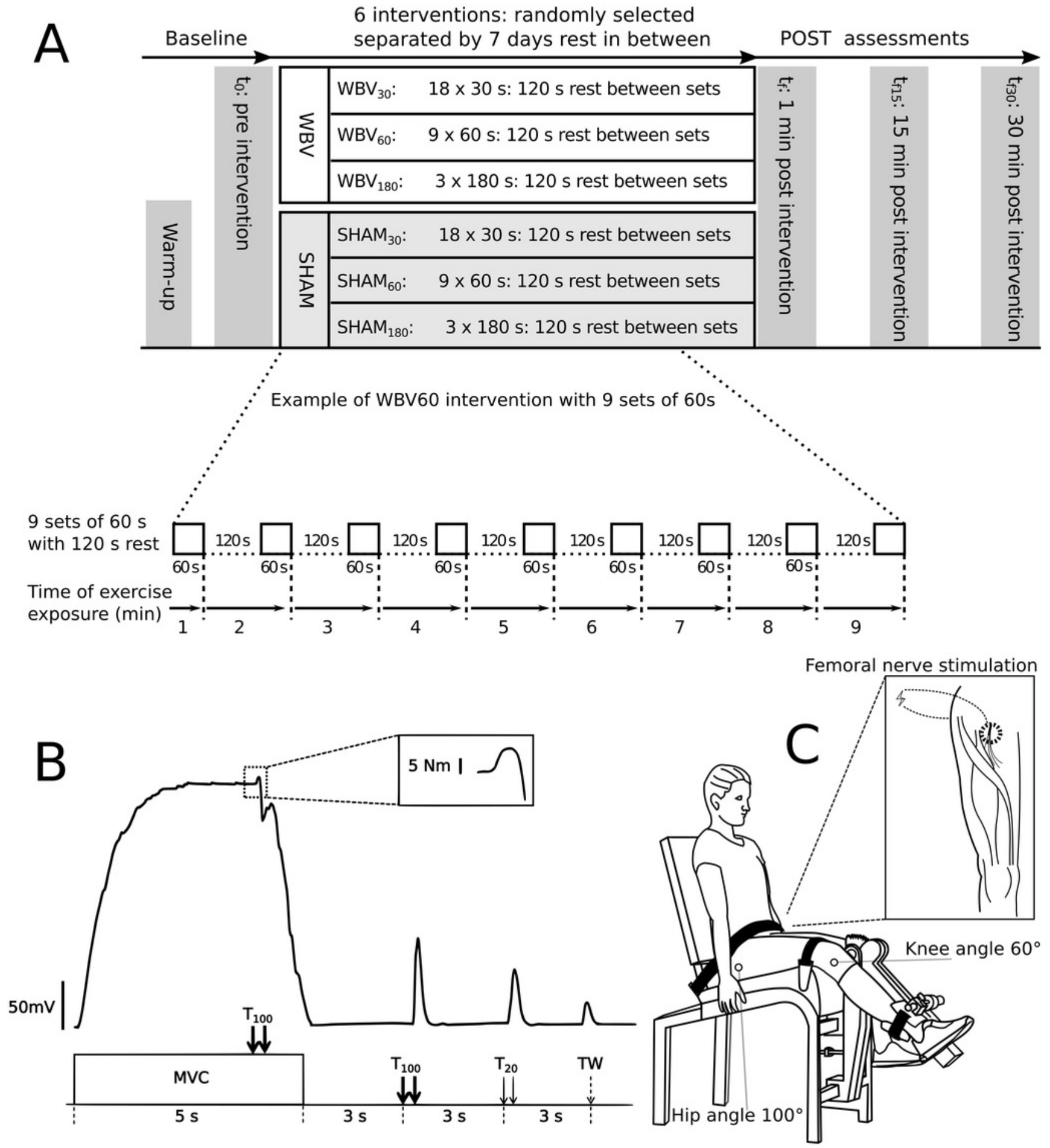
Figure 2

Relative changes from baseline.

(A) Maximum voluntary contraction (MVC), (B) level of voluntary activation (\%VA) and (C) single twitch peak torque (TW ${ }_{\mathrm{PT}}$ ) for WBV (connected black triangles) and SHAM (connected white circles) for trials with different set durations ( $30 \mathrm{~s}, 60 \mathrm{~s}$ and $180 \mathrm{~s}$ ). Values are expressed as mean and standard errors. Black triangles represent statistically significant WBV differences from baseline ( $\Delta \mathbf{\Delta} \mathrm{p}<0.001 ; \boldsymbol{\Lambda} \boldsymbol{p}<0.01 ; \boldsymbol{\Delta} p<0.05$ ). White circles represent statistically significant SHAM differences from baseline (O०Op<0.001; O० $p<$ $0.01 ; O p<0.05)$. 

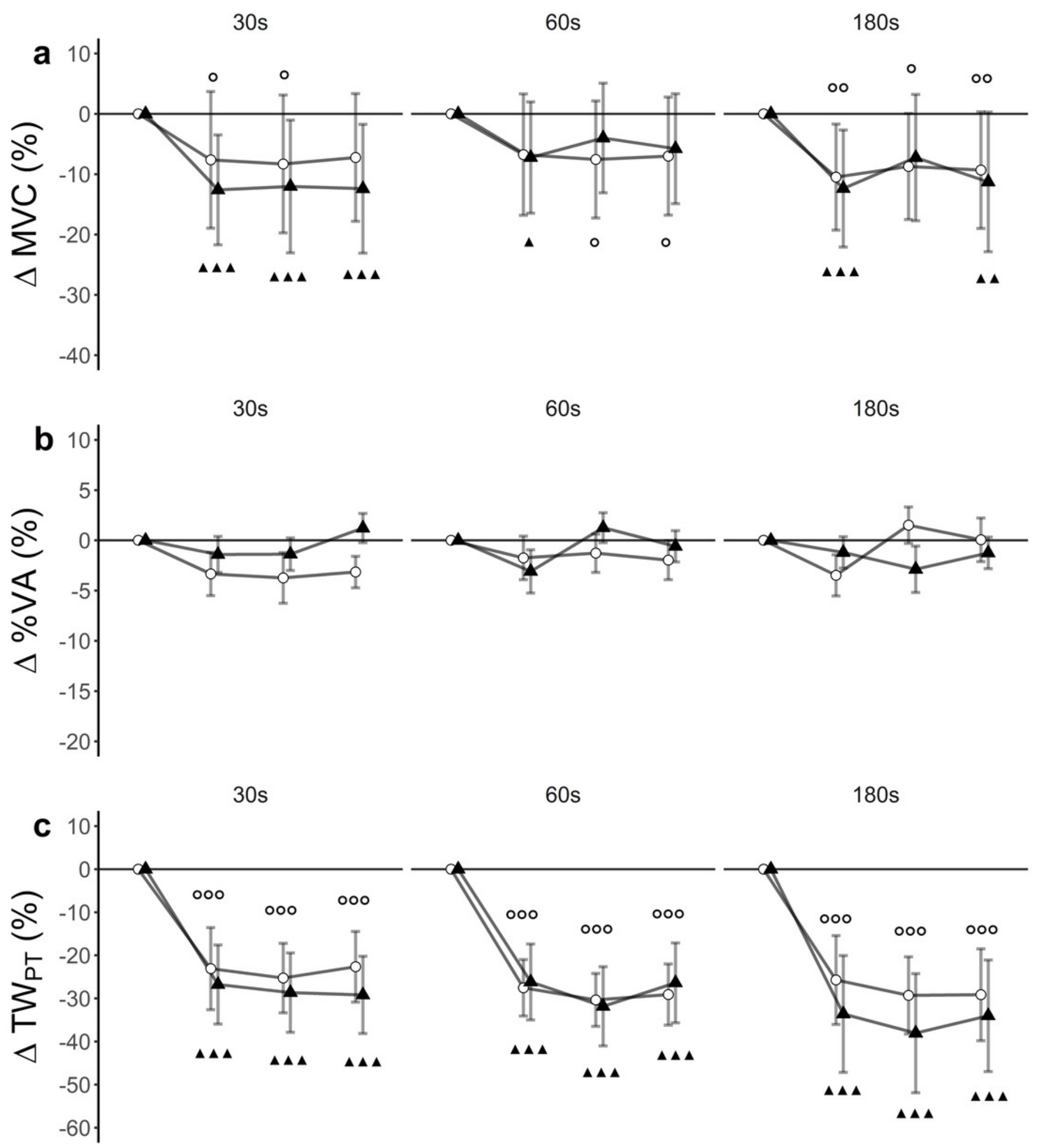


\section{Figure 3}

Relative changes from baseline.

(A) low-frequency doublet $\left(T_{20}\right)$, (B) high-frequency doublet $\left(T_{100}\right)$ and $(C)$ low-high torque frequency ratio $\left(T_{201100}\right)$ for WBV (connected black triangles) and SHAM (connected white circles) for trials with different set durations ( $30 \mathrm{~s}, 60 \mathrm{~s}$ and $180 \mathrm{~s}$ ). Values are expressed as mean and standard errors. Black triangles represent statistically significant WBV differences from baseline ( $\Delta \Lambda \Delta p<0.001 ; \Delta \Lambda p<0.01 ; \Delta p<0.05)$. White circles represent statistically significant SHAM differences from baseline (O०० $p<0.001 ; \bigcirc \bigcirc p<0.01 ; \bigcirc p<0.05)$. 


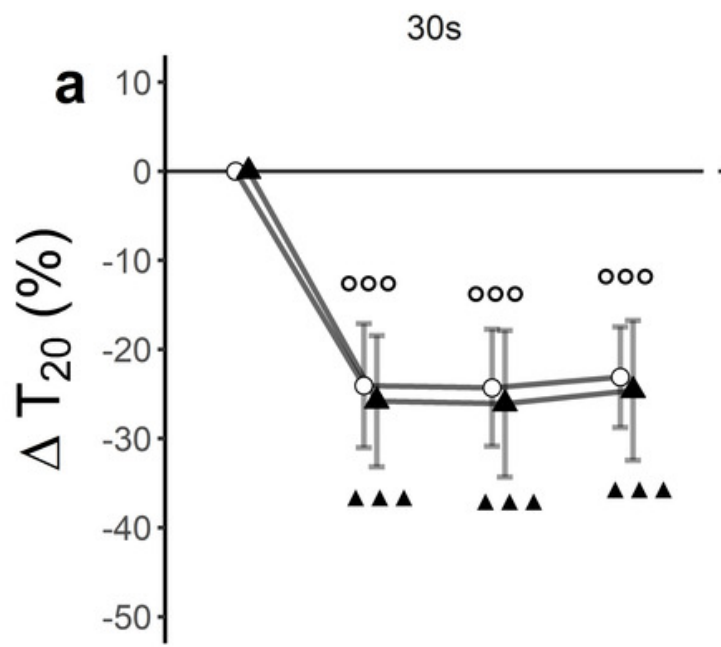

$30 s$

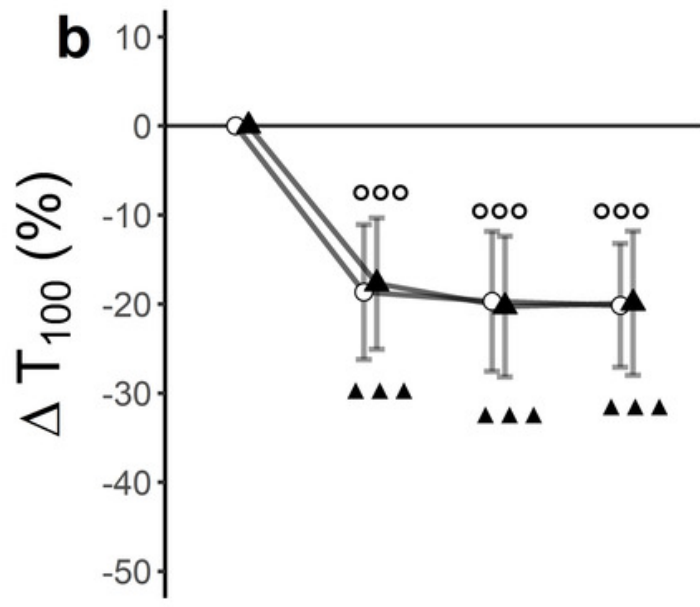

30 s

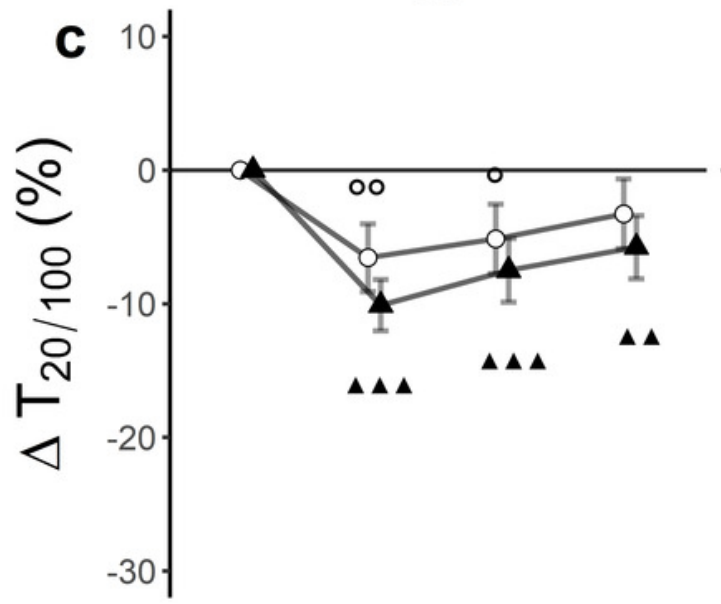

$60 s$

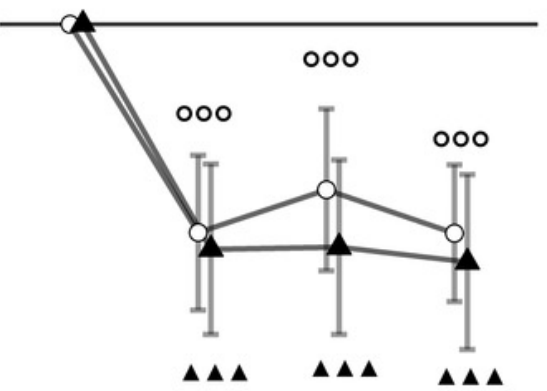

$60 s$
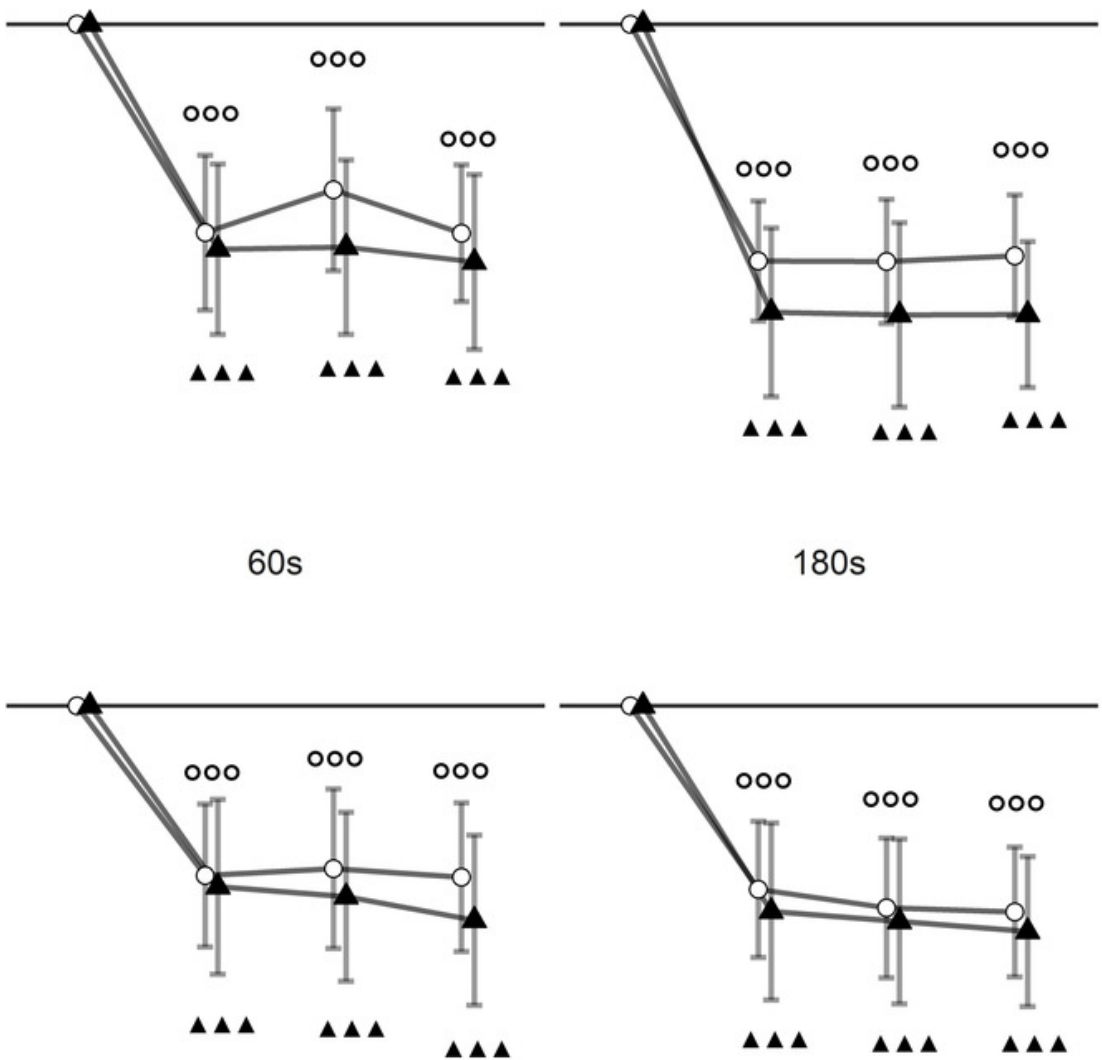

$60 s$

180s

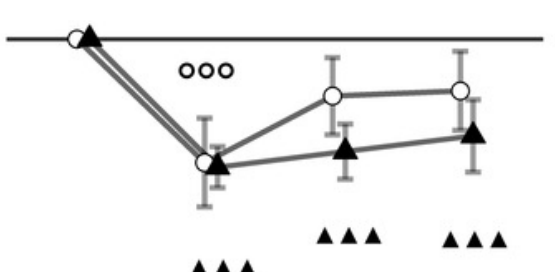

$\boldsymbol{\Lambda \Lambda \Lambda}$

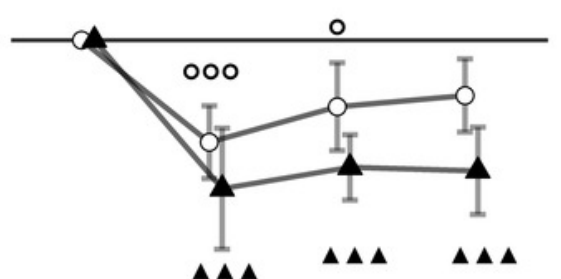




\section{Table $\mathbf{1}$ (on next page)}

Descriptive statistics (mean and SD), within trial relative change from baseline and Cohen $d$ effects size for MVC and \%VA.

$t_{0}$, baseline; $t_{f}$, after intervention; $t_{f 15}, 15$ minutes after intervention; $t_{f 30}, 30$ minutes after intervention. 


\begin{tabular}{|c|c|c|c|c|c|c|c|c|c|c|}
\hline & \multirow{2}{*}{$\begin{array}{c}t_{0} \\
\text { mean (SD) }\end{array}$} & \multicolumn{3}{|c|}{$t_{f}$} & \multicolumn{3}{|c|}{$t_{f 15}$} & \multicolumn{3}{|c|}{$t_{f 30}$} \\
\hline & & mean (SD) & $\Delta \%$ & Cohen $d[95 \% \mathrm{Cl}]$ & mean (SD) & $\Delta \%$ & Cohen $d[95 \% \mathrm{Cl}]$ & mean (SD) & $\Delta \%$ & Cohen $d[95 \% \mathrm{Cl}]$ \\
\hline \multicolumn{11}{|c|}{ Maximum voluntary contraction (MVC) } \\
\hline $\mathrm{SHAM}_{30}$ & $206.04(67.09)$ & $190.31(68.15)$ & -7.63 & $-0.21[-0.31,-0.11]$ & $188.93(68.26)$ & -8.30 & $-0.23[-0.37,-0.09]$ & $191.15(63.97)$ & -7.23 & $-0.21[-0.35,-0.06]$ \\
\hline $\mathrm{WBV}_{30}$ & $219.46(64.63)$ & $191.82(55.25)$ & -12.59 & $-0.42[-0.66,-0.17]$ & $193.06(67.16)$ & -12.03 & $-0.36[-0.54,-0.18]$ & $192.23(64.92)$ & -12.41 & $-0.38[-0.54,-0.23]$ \\
\hline $\mathrm{SHAM}_{60}$ & $215.61(64.31)$ & 201.05 (63.94) & -6.75 & $-0.21[-0.43,0.02]$ & $199.34(61.18)$ & -7.55 & $-0.23[-0.44,-0.03]$ & $200.52(62.06)$ & -7.00 & $-0.22[-0.45,0.01]$ \\
\hline $\mathrm{WBV}_{60}$ & $209.75(63.53)$ & $194.58(56.78)$ & -7.23 & $-0.23[-0.46,0.00]$ & $201.37(57.87)$ & -3.99 & $-0.12[-0.34,0.09]$ & $197.64(56.89)$ & -5.77 & $-0.18[-0.38,0.01]$ \\
\hline $\mathrm{SHAM}_{180}$ & $207.81(62.38)$ & $186.06(51.73)$ & -10.47 & $-0.34[-0.53,-0.16]$ & $189.68(52.72)$ & -8.72 & $-0.28[-0.49,-0.08]$ & $188.45(57.65)$ & -9.32 & $-0.29[-0.47,-0.11]$ \\
\hline $\mathrm{WBV}_{180}$ & $200.36(62.85)$ & $175.58(53.91)$ & -12.37 & $-0.38[-0.68,-0.09]$ & $185.85(61.52)$ & -7.24 & $-0.21[-0.44,0.02]$ & $177.76(65.08)$ & -11.28 & $-0.32[-0.66,0.02]$ \\
\hline \multicolumn{11}{|c|}{ Level of voluntary activation (\%VA) } \\
\hline $\mathrm{SHAM}_{30}$ & $93.05(3.00)$ & $89.94(6.14)$ & -3.34 & $-0.58[-1.22,0.05]$ & $89.57(7.14)$ & -3.74 & $-0.58[-1.34,0.19]$ & $90.12(4.47)$ & -3.15 & $-0.40[-0.72,0.52]$ \\
\hline $\mathrm{WBV}_{30}$ & $90.74(3.98)$ & $89.47(5.07)$ & -1.41 & $-0.25[-0.97,0.46]$ & $89.49(4.58)$ & -1.38 & $-0.26[-0.87,0.34]$ & $91.85(4.21)$ & 1.22 & $0.25[-0.39,0.88]$ \\
\hline $\mathrm{SHAM}_{60}$ & $89.27(4.78)$ & $87.71(6.00)$ & -1.75 & $-0.26[-0.78,0.26]$ & $88.14(5.32)$ & -1.27 & $-0.20[-0.74,0.34]$ & $87.52(5.39)$ & -1.96 & $-0.31[-0.85,0.23]$ \\
\hline $\mathrm{WBV}_{60}$ & $90.20(4.85)$ & $87.41(5.96)$ & -3.09 & $-0.31[-0.70,0.55]$ & $91.33(4.29)$ & 1.26 & $0.17[-0.59,0.67]$ & $89.66(4.41)$ & -0.59 & $-0.03[-0.64,0.62]$ \\
\hline $\mathrm{SHAM}_{180}$ & $87.40(6.82)$ & $84.36(5.47)$ & -3.47 & $-0.45[-1.08,0.19]$ & $88.73(5.06)$ & 1.52 & $0.20[-0.55,0.95]$ & $87.45(6.01)$ & 0.05 & $0.01[-0.75,0.77]$ \\
\hline$W_{B} V_{180}$ & $88.54(5.36)$ & $87.48(4.31)$ & -1.20 & $-0.17[-0.67,0.59]$ & $86.00(6.28)$ & -2.87 & $-0.40[-1.03,0.24]$ & $87.43(4.33)$ & -1.25 & $-0.21[-0.98,0.57]$ \\
\hline
\end{tabular}




\section{Table 2 (on next page)}

Descriptive statistics (mean and SD), within trial relative change from baseline and Cohen $d$ effects size for $T_{20}, T_{100}, T_{20 / 100}$ and $T W_{P T}$.

$t_{0}$, baseline; $t_{f}$, after intervention; $t_{115}, 15$ minutes after intervention; $t_{130}, 30$ minutes after intervention. 


\begin{tabular}{|c|c|c|c|c|c|c|c|c|c|c|}
\hline & \multirow{2}{*}{$\begin{array}{c}t_{0} \\
\text { mean (SD) }\end{array}$} & \multicolumn{4}{|c|}{$t_{f}$} & \multicolumn{2}{|r|}{$t_{f 15}$} & \multicolumn{3}{|c|}{$t_{f 30}$} \\
\hline & & mean (SD) & $\Delta \%$ & Cohen $d[95 \% \mathrm{Cl}]$ & mean (SD) & $\Delta \%$ & Cohen d $[95 \% \mathrm{Cl}]$ & mean (SD) & $\Delta \%$ & Cohen d $[95 \% \mathrm{Cl}]$ \\
\hline \multicolumn{11}{|c|}{ Low-frequency doublet $\left(T_{20}\right)$} \\
\hline $\mathrm{SHAM}_{30}$ & $75.26(18.37)$ & $57.14(12.56)$ & -24.07 & $-1.04[-1.51,-0.58]$ & $56.97(11.84)$ & -24.30 & $-0.63[-0.78,0.42]$ & $57.87(10.33)$ & -23.11 & $-0.63[-0.78,0.42]$ \\
\hline $\mathrm{WBV}_{30}$ & $80.47(20.30)$ & $59.70(13.91)$ & -25.80 & $-1.08[-1.43,-0.73]$ & $59.47(15.47)$ & -26.10 & $-1.05[-1.39,-0.72]$ & $60.67(15.04)$ & -24.61 & $-0.63[-0.78,0.42]$ \\
\hline $\mathrm{SHAM}_{60}$ & $82.03(22.79)$ & $62.84(17.27)$ & -23.39 & $-0.86[-1.45,-0.27]$ & $66.78(19.18)$ & -18.59 & $-0.66[-1.34,0.03]$ & $62.78(15.23)$ & -23.46 & $-0.90[-1.39,-0.41]$ \\
\hline $\mathrm{WBV}_{60}$ & $76.11(18.34)$ & $56.88(17.20)$ & -25.26 & $-0.98[-1.44,-0.52]$ & $57.06(17.68)$ & -25.03 & $-0.96[-1.34,-0.58]$ & $55.79(17.33)$ & -26.70 & $-1.03[-1.52,-0.54]$ \\
\hline SHAM $_{180}$ & $82.24(17.44)$ & $60.38(12.87)$ & -26.58 & $-0.63[-0.78,0.42]$ & $60.35(13.30)$ & -26.62 & $-0.63[-0.78,0.42]$ & $60.86(13.15)$ & -26.00 & $-0.63[-0.78,0.42]$ \\
\hline $\mathrm{WBV}_{180}$ & $79.59(18.84)$ & $53.86(16.14)$ & -32.33 & $-1.33[-1.69,-0.97]$ & $53.62(17.56)$ & -32.63 & $-1.29[-1.68,-0.90]$ & $53.66(13.88)$ & -32.58 & $-1.42[-1.82,-1.02]$ \\
\hline \multicolumn{11}{|c|}{ High-frequency doublet $\left(T_{100}\right)$} \\
\hline $\mathrm{SHAM}_{30}$ & $78.56(20.46)$ & $63.90(15.30)$ & -18.66 & $-0.74[-1.02,-0.45]$ & $63.09(15.68)$ & -19.69 & $-0.77[-1.02,-0.52]$ & $62.73(13.77)$ & -20.15 & $-0.82[-1.10,-0.54]$ \\
\hline $\mathrm{WBV}_{30}$ & $82.52(21.65)$ & $67.91(15.83)$ & -17.71 & $-0.70[-0.94,-0.45]$ & $65.79(16.42)$ & -20.28 & $-0.79[-1.02,-0.56]$ & $66.10(16.90)$ & -19.90 & $-0.63[-0.78,0.42]$ \\
\hline $\mathrm{SHAM}_{60}$ & $87.98(22.21)$ & $71.22(18.06)$ & -19.05 & $-0.75[-1.04,-0.46]$ & $71.91(20.34)$ & -18.27 & $-0.68[-1.04,-0.33]$ & $71.07(18.78)$ & -19.23 & $-0.75[-1.08,-0.41]$ \\
\hline $\mathrm{WBV}_{60}$ & $81.00(19.98)$ & $64.55(20.02)$ & -20.30 & $-0.75[-1.32,-0.17]$ & $63.64(19.06)$ & -21.43 & $-0.81[-1.36,-0.25]$ & $61.50(18.58)$ & -24.06 & $-0.92[-1.57,-0.26]$ \\
\hline SHAM $_{180}$ & $88.05(21.36)$ & $69.93(16.90)$ & -20.58 & $-0.85[-1.18,-0.52]$ & $68.09(16.89)$ & -22.68 & $-0.94[-1.28,-0.60]$ & $67.69(15.60)$ & -23.12 & $-0.99[-1.39,-0.59]$ \\
\hline $\mathrm{WBV}_{180}$ & $83.10(21.07)$ & $63.93(20.07)$ & -23.07 & $-0.84[-1.10,-0.59]$ & $62.99(18.42)$ & -24.20 & $-0.92[-1.20,-0.64]$ & $62.06(16.54)$ & -25.33 & $-1.01[-1.30,-0.71]$ \\
\hline \multicolumn{11}{|c|}{ Low- to high-frequency doublet ration $\left(T_{20 / 100}\right)$} \\
\hline $\mathrm{SHAM}_{30}$ & $0.96(0.06)$ & $0.90(0.07)$ & -6.56 & $-0.89[-1.45,-0.32]$ & $0.91(0.08)$ & -5.15 & $-0.68[-1.09,-0.27]$ & $0.93(0.08)$ & -3.27 & $-0.43[-0.78,-0.07]$ \\
\hline $\mathrm{WBV}_{30}$ & $0.97(0.05)$ & $0.88(0.05)$ & -10.11 & $-0.60[-0.77,0.43]$ & $0.90(0.07)$ & -7.50 & $-0.49[-0.74,0.48]$ & $0.92(0.07)$ & -5.77 & $-0.44[-0.73,0.50]$ \\
\hline $\mathrm{SHAM}_{60}$ & $0.97(0.07)$ & $0.88(0.09)$ & -9.24 & $-0.54[-0.75,0.46]$ & $0.93(0.08)$ & -4.27 & $-0.42[-0.73,0.51]$ & $0.94(0.09)$ & -3.87 & $-0.35[-0.71,0.53]$ \\
\hline $\mathrm{WBV}_{60}$ & $0.98(0.06)$ & $0.89(0.04)$ & -9.58 & $-1.56[-2.23,-0.89]$ & $0.90(0.06)$ & -8.42 & $-1.22[-1.86,-0.57]$ & $0.91(0.08)$ & -7.24 & $-0.90[-1.78,-0.02]$ \\
\hline $\mathrm{SHAM}_{180}$ & $0.96(0.08)$ & $0.88(0.08)$ & -7.64 & $-0.83[-1.28,-0.38]$ & $0.91(0.09)$ & -4.98 & $-0.49[-0.82,-0.15]$ & $0.92(0.08)$ & -4.15 & $-0.44[-0.80,-0.08]$ \\
\hline$W_{B V} V_{180}$ & $0.98(0.06)$ & $0.87(0.12)$ & -11.12 & $-1.00[-1.59,-0.40]$ & $0.88(0.07)$ & -9.53 & $-1.28[-1.80,-0.75]$ & $0.88(0.09)$ & -9.78 & $-1.10[-1.54,-0.67]$ \\
\hline \multicolumn{11}{|c|}{ Single twitch peak torque ( $\left.T W_{P T}\right)$} \\
\hline $\mathrm{SHAM}_{30}$ & $26.81(7.53)$ & $20.62(6.21)$ & -23.10 & $-0.63[-0.78,0.42]$ & $20.04(5.12)$ & -25.28 & $-0.63[-0.78,0.42]$ & $20.74(5.39)$ & -22.65 & $-0.84[-1.29,-0.40]$ \\
\hline $\mathrm{WBV}_{30}$ & $26.62(8.07)$ & $19.50(5.65)$ & -26.77 & $-0.63[-0.78,0.42]$ & $19.00(5.53)$ & -28.64 & $-1.00[-1.36,-0.64]$ & $18.85(5.34)$ & -29.19 & $-1.03[-1.42,-0.64]$ \\
\hline $\mathrm{SHAM}_{60}$ & $27.37(8.27)$ & $19.83(4.11)$ & -27.54 & $-1.05[-1.53,-0.56]$ & $19.06(3.70)$ & -30.35 & $-1.18[-1.81,-0.54]$ & $19.41(4.35)$ & -29.10 & $-1.09[-1.58,-0.60]$ \\
\hline$W_{B V} V_{60}$ & $26.77(8.11)$ & $19.76(5.50)$ & -26.17 & $-0.92[-1.26,-0.57]$ & $18.25(5.31)$ & -31.84 & $-0.63[-0.78,0.42]$ & $19.71(5.78)$ & -26.37 & $-0.91[-1.38,-0.44]$ \\
\hline $\mathrm{SHAM}_{180}$ & $26.72(7.68)$ & $19.85(6.47)$ & -25.72 & $-0.88[-1.09,-0.67]$ & $18.90(5.35)$ & -29.29 & $-1.07[-1.36,-0.78]$ & $18.94(6.39)$ & -29.15 & $-0.63[-0.78,0.42]$ \\
\hline $\mathrm{WBV}_{180}$ & $27.20(8.23)$ & $18.06(7.74)$ & -33.60 & $-1.04[-1.32,-0.75]$ & $16.85(7.37)$ & -38.06 & $-1.20[-1.58,-0.82]$ & $17.95(7.35)$ & -34.01 & $-1.07[-1.43,-0.72]$ \\
\hline
\end{tabular}

1 


\section{Table 3(on next page)}

Within-trial Tukey corrected t-test comparison with baseline.

Asterisks represent statistically significant differences from baseline (*** $p<0.001$; $* * p<$ $0.01 ; * p<0.05) . t_{0}$, baseline; $t_{f}$, after intervention; $t_{f 15}, 15$ minutes after intervention; $t_{f 30}, 30$ minutes after intervention. 


\begin{tabular}{|c|c|c|c|c|c|c|c|c|c|}
\hline \multirow[b]{2}{*}{ visit } & \multicolumn{3}{|c|}{$t_{f}$} & \multicolumn{3}{|c|}{$t_{f 15}$} & \multicolumn{3}{|c|}{$t_{f 30}$} \\
\hline & t-value & p-value & sig. & t-value & p-value & sig. & t-value & p-value & sig. \\
\hline \multicolumn{10}{|c|}{ Maximum voluntary contraction (MVC) } \\
\hline $\mathrm{SHAM}_{30}$ & -2.53 & 0.04 & * & -2.75 & 0.02 & * & -2.40 & 0.05 & \\
\hline $\mathrm{WBV}_{30}$ & -4.45 & $<0.001$ & $* * *$ & -4.25 & $<0.001$ & $* * *$ & -4.38 & $<0.001$ & *** \\
\hline $\mathrm{SHAM}_{60}$ & -2.34 & 0.06 & & -2.62 & 0.03 & * & -2.43 & 0.05 & * \\
\hline $\mathrm{WBV}_{60}$ & -2.44 & 0.05 & * & -1.35 & 0.45 & & -1.95 & 0.15 & \\
\hline $\mathrm{SHAM}_{180}$ & -3.50 & 0.002 & $* *$ & -2.92 & 0.01 & * & -3.12 & 0.006 & ** \\
\hline $\mathrm{WBV}_{180}$ & -3.99 & $<0.001$ & $* * *$ & -2.34 & 0.06 & & -3.64 & 0.001 & ** \\
\hline \multicolumn{10}{|c|}{ Level of voluntary activation (\%VA) } \\
\hline $\mathrm{SHAM}_{30}$ & -2.02 & 0.13 & & -2.26 & 0.07 & & -1.90 & 0.17 & \\
\hline $\mathrm{WBV}_{30}$ & -0.83 & 0.79 & & -0.81 & 0.80 & & 0.72 & 0.85 & \\
\hline $\mathrm{SHAM}_{60}$ & -1.01 & 0.68 & & -0.74 & 0.84 & & -1.14 & 0.59 & \\
\hline $\mathrm{WBV}_{60}$ & -1.81 & 0.20 & & 0.74 & 0.84 & & -0.35 & 0.98 & \\
\hline SHAM $_{180}$ & -1.97 & 0.14 & & 0.86 & 0.77 & & 0.03 & 1.00 & \\
\hline$W_{B} V_{180}$ & -0.69 & 0.87 & & -1.65 & 0.27 & & -0.72 & 0.85 & \\
\hline \multicolumn{10}{|c|}{ Low-frequency doublet $\left(T_{20}\right)$} \\
\hline $\mathrm{SHAM}_{30}$ & -6.08 & $<0.001$ & $* * *$ & -6.14 & $<0.001$ & $* * *$ & -5.84 & $<0.001$ & *** \\
\hline $\mathrm{WBV}_{30}$ & -6.97 & $<0.001$ & $* * *$ & -7.05 & $<0.001$ & $* * *$ & -6.65 & $<0.001$ & *** \\
\hline $\mathrm{SHAM}_{60}$ & -6.44 & $<0.001$ & $* * *$ & -5.12 & $<0.001$ & $* * *$ & -6.46 & $<0.001$ & *** \\
\hline $\mathrm{WBV}_{60}$ & -6.46 & $<0.001$ & $* * *$ & -6.40 & $<0.001$ & $* * *$ & -6.82 & $<0.001$ & $* * *$ \\
\hline SHAM $_{180}$ & -7.34 & $<0.001$ & $* * *$ & -7.35 & $<0.001$ & $* * *$ & -7.18 & $<0.001$ & *** \\
\hline$W_{B} V_{180}$ & -8.64 & $<0.001$ & $* * *$ & -8.72 & $<0.001$ & $* * *$ & -8.71 & $<0.001$ & $* * *$ \\
\hline \multicolumn{10}{|c|}{ High-frequency doublet $\left(T_{100}\right)$} \\
\hline $\mathrm{SHAM}_{30}$ & -5.42 & $<0.001$ & $* * *$ & -5.71 & $<0.001$ & $* * *$ & -5.85 & $<0.001$ & *** \\
\hline $\mathrm{WBV}_{30}$ & -5.40 & $<0.001$ & $* * *$ & -6.18 & $<0.001$ & $* * *$ & -6.07 & $<0.001$ & *** \\
\hline $\mathrm{SHAM}_{60}$ & -6.19 & $<0.001$ & $* * *$ & -5.94 & $<0.001$ & $\star * \star$ & -6.25 & $<0.001$ & *** \\
\hline $\mathrm{WBV}_{60}$ & -6.08 & $<0.001$ & $* * *$ & -6.41 & $<0.001$ & $\star * *$ & -7.20 & $<0.001$ & *** \\
\hline SHAM $_{180}$ & -6.70 & $<0.001$ & $* * *$ & -7.38 & $<0.001$ & $* * *$ & -7.52 & $<0.001$ & $* * *$ \\
\hline $\mathrm{WBV}_{180}$ & -7.08 & $<0.001$ & $* * *$ & -7.43 & $<0.001$ & $* * *$ & -7.78 & $<0.001$ & *** \\
\hline \multicolumn{10}{|c|}{ Low- to high-frequency doublet ration $\left(T_{20 / 100}\right)$} \\
\hline $\mathrm{SHAM}_{30}$ & -3.57 & 0.001 & ** & -2.81 & 0.02 & * & -1.78 & 0.21 & \\
\hline $\mathrm{WBV}_{30}$ & -5.57 & $<0.001$ & $* * *$ & -4.13 & $<0.001$ & $* * *$ & -3.18 & 0.005 & ** \\
\hline $\mathrm{SHAM}_{60}$ & -5.09 & $<0.001$ & $* * *$ & -2.35 & 0.06 & & -2.13 & 0.10 & \\
\hline $\mathrm{WBV}_{60}$ & -5.30 & $<0.001$ & $* * *$ & -4.66 & $<0.001$ & $* * *$ & -4.01 & $<0.001$ & *** \\
\hline SHAM $_{180}$ & -4.12 & $<0.001$ & $* * *$ & -2.69 & 0.02 & * & -2.24 & 0.08 & \\
\hline $\mathrm{WBV}_{180}$ & -6.14 & $<0.001$ & $* * *$ & -5.26 & $<0.001$ & $* * *$ & -5.40 & $<0.001$ & *** \\
\hline \multicolumn{10}{|c|}{ Single twitch peak torque (TW } \\
\hline $\mathrm{SHAM}_{30}$ & -5.70 & $<0.001$ & $* * *$ & -6.24 & $<0.001$ & $* * *$ & -5.59 & $<0.001$ & $* * *$ \\
\hline $\mathrm{WBV}_{30}$ & -6.56 & $<0.001$ & $* * *$ & -7.01 & $<0.001$ & $* * *$ & -7.15 & $<0.001$ & *** \\
\hline SHAM $_{60}$ & -6.93 & $<0.001$ & $* * *$ & -7.64 & $<0.001$ & $* * *$ & -7.33 & $<0.001$ & *** \\
\hline $\mathrm{WBV}_{60}$ & -6.44 & $<0.001$ & $* * *$ & -7.84 & $<0.001$ & $* * *$ & -6.49 & $<0.001$ & $* * *$ \\
\hline SHAM $_{180}$ & -6.32 & $<0.001$ & $* * *$ & -7.20 & $<0.001$ & $* * *$ & -7.17 & $<0.001$ & $* * *$ \\
\hline $\mathrm{WBV}_{180}$ & -8.41 & $<0.001$ & $* * *$ & -9.52 & $<0.001$ & $* * *$ & -8.51 & $<0.001$ & $* * *$ \\
\hline
\end{tabular}

